\title{
Rapid and Intermittent Cotransport of Slow Component-b Proteins
}

\author{
Subhojit Roy, ${ }^{1,2}$ Matthew J. Winton, ${ }^{1}$ Mark M. Black, ${ }^{4}$ John Q. Trojanowski, ${ }^{1,2,3}$ and Virginia M.-Y. Lee ${ }^{1,2,3}$ \\ ${ }^{1}$ Center for Neurodegenerative Disease Research, ${ }^{2}$ Department of Pathology and Laboratory Medicine, and ${ }^{3}$ Institute on Aging, University of Pennsylvania \\ School of Medicine, Philadelphia, Pennsylvania 19104, and ${ }^{4}$ Department of Anatomy and Cell Biology, Temple University Hospital, Philadelphia, \\ Pennsylvania 19130
}

\begin{abstract}
After synthesis in neuronal perikarya, proteins destined for synapses and other distant axonal sites are transported in three major groups that differ in average velocity and protein composition: fast component (FC), slow component-a (SCa), and slow component-b (SCb). The FC transports mainly vesicular cargoes at average rates of $\sim 200-400 \mathrm{~mm} / \mathrm{d}$. SCa transports microtubules and neurofilaments at average rates of $\sim 0.2-1 \mathrm{~mm} / \mathrm{d}$, whereas SCb translocates $\sim 200$ diverse proteins critical for axonal growth, regeneration, and synaptic function at average rates of $\sim 2-8 \mathrm{~mm} / \mathrm{d}$. Several neurodegenerative diseases are characterized by abnormalities in one or more SCb proteins, but little is known about mechanisms underlying SCb compared with FC and SCa. Here, we use live-cell imaging to visualize and quantify the axonal transport of three SCb proteins, $\alpha$-synuclein, synapsin-I, and glyceraldehyde-3-phosphate dehydrogenase in cultured hippocampal neurons, and directly compare their transport to synaptophysin, a prototypical FC protein. All three SCb proteins move rapidly but infrequently with pauses during transit, unlike synaptophysin, which moves much more frequently and persistently. By simultaneously visualizing the transport of proteins at high temporal and spatial resolution, we show that the dynamics of $\alpha$-synuclein transport are distinct from those of synaptophysin but similar to other SCb proteins. Our observations of the cotransport of multiple SCb proteins in single axons suggest that they move as multiprotein complexes. These studies offer novel mechanistic insights into SCb and provide tools for further investigating its role in disease processes.
\end{abstract}

Key words: axonal transport; slow transport; slow component-b; $\alpha$-synuclein; synaptophysin; protein complexes

\section{Introduction}

Proteins synthesized in the neuronal cell body are delivered to distant sites in axons, including synapses, by axonal transport, an energy-dependent process that occurs throughout the life of the neuron. Materials are delivered to the axon in three major components termed fast component (FC), slow component-a (SCa), and slow component-b (SCb). Significant progress has been made in understanding FC and SCa, and many mechanistic details of FC and SCa transport have been defined, primarily because of development of methods to visualize their transport in axons of living neurons (Brown, 2003; Baas and Buster, 2004). In contrast to $\mathrm{FC}$ and $\mathrm{SCa}$, mechanisms underlying $\mathrm{SCb}$ remain enigmatic.

Early studies using radio-isotopic labeling methods showed

Received Nov. 17, 2006; revised Jan. 9, 2007; accepted Feb. 6, 2007.

This work was supported by National Institute on Aging Grant AG09215 and by a grant from the Picower Foundation and the Benaroyo Family. S.R. is supported by a postdoctoral grant from the American Parkinson's Disease Association. We thank Chi Li for his technical expertise with the CDNA constructs, Dr. C. Kaether (LudwigMaximilians-University, München, Germany) for the synaptophysin::GFP construct, Dr. H. T. Kao (Rockefeller University, New York, NY) for the synapsin promoter construct, Dr. G. Augustine (Duke University, Durham, NC) for the GFP::synapsin-I construct, and Dr. J. Bereiter-Hahn (Kinematic Cell Research Group, Goethe University, Germany) for the GFP::GAPDH construct.

Correspondence should be addressed to Dr. Virginia M.-Y. Lee, Center for Neurodegenerative Disease Research, Department of Pathology and Laboratory Medicine, University of Pennsylvania School of Medicine, Maloney Building, 3rd Floor, 3600 Spruce Street, Philadelphia, PA 19104. E-mail: vmylee@mail.med.upenn.edu.

D0I:10.1523/JNEUROSCI.4999-06.2007

Copyright $\odot 2007$ Society for Neuroscience $\quad$ 0270-6474/07/273131-08\$15.00/0 that $\mathrm{SCa}$ and $\mathrm{SCb}$ are two distinct slow components. Although both move at slow rates, overall kinetics and the individual polypeptide composition of SCb is primarily different from SCa (Black and Lasek, 1980). While SCa transports the major cytoskeletal structures (i.e., neurofilaments and microtubules), SCb transports $>200$ diverse proteins that are key components of axons and synapses. Average rates of SCb are also $\sim 2-10$ times faster than SCa. Only a handful of SCb proteins have been identified, and they are enormously diverse, including enzymes such as glyceraldehyde-3-phosphate dehydrogenase (GAPDH) and superoxide dismutase-1; various chaperones calmodulin and actin-depolymerizing factor; presynaptic proteins such as synap$\sin , \alpha$-synuclein, and clathrin; motor proteins such as dynein and myosin; and cytoskeletal proteins such as actin and spectrin (Garner and Lasek, 1982; Lasek et al., 1984; Baitinger and Willard, 1987; Bray et al., 1992; Dillman et al., 1996; Yuan et al., 1999; Bourke et al., 2002). The importance of SCb for neuronal viability is highlighted by studies showing that several SCb proteins, including dynein (Hafezparast et al., 2003), superoxide dismutase-1 (Borchelt et al., 1998), and $\alpha$-synuclein (Jensen et al., 1999; Forman et al., 2004; Saha et al., 2004), are linked mechanistically to neurodegenerative diseases.

Several in vivo radiolabeling transport studies have specifically implicated SCb in axon growth and regeneration. One of the first indications was the similarity in the average rates of $\mathrm{SCb}$ and axonal regeneration (Wujek and Lasek, 1983), raising the possi- 
bility that SCb provides materials essential for axon growth and that their delivery by SCb influences axonal growth. This was reinforced by subsequent studies showing that manipulations accelerating SCb transport also accelerated regeneration (Maier and McQuarrie, 1990; Jacob and McQuarrie, 1991), and by the demonstration that proteins critical to normal growth cone motility and guidance such as dynein, actin, and myosin were transported in SCb (Baas and Buster, 2004).

Despite observations linking SCb to axon growth, neuronal homeostasis, and neurodegenerative diseases, fundamental mechanisms of SCb transport are unknown. Almost all available information on SCb was obtained using radio-isotopic labeling methods, but these techniques lack the spatial and temporal resolution needed to dissect transport mechanisms. To address this issue, we quantitatively analyzed the transport of three fluorescently tagged $\mathrm{SCb}$ proteins, $\alpha$-synuclein, synapsin-I, and GAPDH, and an FC protein synaptophysin in axons of living neurons. Our studies combine conventional time-lapse imaging with a novel approach that enabled us to simultaneously visualize transport of multiple proteins in the same axon. The results reveal fundamental aspects of SCb transport.

\section{Materials and Methods}

Antibodies and plasmid constructs. For immunofluorescence studies of $\alpha$-synuclein and synaptophysin, affinity-purified rabbit polyclonal (SNL-1) and mouse monoclonal (clone SY38; Chemicon, Temecula, CA) were used, respectively. Using standard molecular biology protocols, full-length $\alpha$-synuclein driven by cytomegalovirus (CMV) promoter was tagged at the $\mathrm{N}$ terminus with monomeric red fluorescent protein (mRFP) (mRFP:: $\alpha$-SYN) or green fluorescent protein (GFP) (GFP:: $\alpha$-SYN) by subcloning them into the pDsRed monomer $\mathrm{Cl}$ or enhanced GFP (EGFP)-C1 vector, respectively (Clontech, Mountain View, CA). In addition, the CMV promoter in GFP:: $\alpha$-SYN was also replaced with a synapsin-I promoter. The GFP::RhoB (pAcGFP1-Endo) is commercially available (Clontech), and the synaptophysin C-terminal tagged GFP (SYP::GFP) construct has been described previously (Kaether et al., 2000).

Cell culture and transfection. Hippocampal cultures were obtained from brains of embryonic day 18 (E18) to E19 C57Bl/6 mice following standard protocols. Briefly, dissociated cells were plated at a density of 50,000-100,000 cells/cm in poly-D-lysine-coated glass-bottom culture dishes (MatTek, Ashland, MA) and maintained in Neurobasal/B27 media (Invitrogen, Carlsbad, CA) supplemented with $0.5 \mathrm{~mm}$ glutamine. Cells were transfected with the respective construct(s) using Lipofectamine-2000 (Invitrogen) at 7-8 d in vitro (DIV) according to the instructions of the manufacturer, and transport studies were performed on 10-15 DIV neurons. All animal studies were performed in accordance with University of Pennsylvania guidelines.

Microscopy and live-cell imaging. For live-cell imaging, cells growing in $\mathrm{NB} / \mathrm{B} 27$ media were quickly rinsed (four times) with a nutrient-enriched modified L-15-based media that buffers $\mathrm{pH}$ at atmospheric $\mathrm{CO}_{2}$. The modified L-15 media is described by Roy et al. (2000), except glutamate and nerve growth factor were omitted. In the last rinse, the L-15-based medium was supplemented with Oxyrase (1:100; Oxyrase, Mansfield, $\mathrm{OH})$ to minimize photodamage during fluorescence imaging. A sealed chamber containing the L-15/Oxyrase media was then created on the MatTek (Ashland, MA) culture dishes using a coverslip and a siliconebased sealant (Compound 111; Dow Corning, Midland, MI). Cells were then transferred onto a custom-built Plexiglas incubator housed over a Nikon TE-2000-E (Nikon, Tokyo, Japan) inverted epifluorescence microscope. The incubator was kept at $\sim 35^{\circ} \mathrm{C}$ using an Air-Therm ATX temperature controller (World Precision Instruments, Sarasota, FL). The $100 \times$ oil immersion lens for imaging was also heated to $\sim 35^{\circ} \mathrm{C}$ using a Bioptechs objective heater (Bioptechs, Butler, PA). To further minimize photodamage and photobleaching, the intensity of the fluorescence lamp was reduced to $\sim 25 \%$ of total intensity using neutral-density filters. Under these conditions, neurons extended growth cones and explored their environment for $1 \mathrm{~h}$ or more. Regions of thin axons away from growth cones, adjacent cell bodies, or dendrites that had minimal synaptic-like varicosities were typically chosen for live imaging. All images were binned $(2 \times 2)$, and exposure times were typically $400-1000$ $\mathrm{ms}$, with the camera operating at maximum gain. For display only, a $3 \times$ 3 low-pass filter was used to reduce background in some movies.

Kymograph analysis. All image acquisition and processing was done using MetaMorph (Molecular Devices, Downingtown, PA). For analyzing images and videos, only the camera noise was subtracted from the videos without additional processing. To avoid objects that moved bidirectionally without net vectorial movement, only particles that moved at least $10 \mu \mathrm{m}$ in any direction were analyzed. Kymographs were generated using the dropdown menu "kymograph" in MetaMorph software. For calculating average velocities of moving particles, lines were traced over the kymographs, and the number of pixels corresponding to the $\mathrm{X}$ and $\mathrm{Y}$ axes of the straight line was obtained using the dropdown menu "region measurements". The "slope" was then calculated from these measurements. For analyzing the SYP::GFP data, representative kymographs were obtained. A subset of 100 frames (frames $200-300, \sim 100$ s imaging time representing $\sim 10 \%$ of each video) was analyzed from every SYP::GFP video. These shorter representative videos were simpler to analyze because they had fewer moving particles. To analyze the proportion of moving versus paused $\alpha$-synuclein/synaptophysin particles, shorter representative kymographs from both mRFP:: $\alpha$-SYN and SYP::GFP live-imaging videos were taken (from frames 200-300, same as above). The ratio of moving/paused particles was then obtained from these relatively simpler kymographs. For drawing the overlay kymographs, lines were independently traced over each of the two kymographs, binarized, and then pseudocolored (red/green). The two binarized images were then merged and transferred to Adobe Photoshop for display (Adobe Systems, San Jose, CA). Dual-cam videos were intentionally shifted for independent visualization and comparison of each channel. For statistical analyses, the two groups of data ( $\alpha$-synuclein and synaptophysin average velocities) were compared using nonparametric tests (Mann-Whitney; two-tailed). The $p$ values for anterograde and retrograde velocities were 0.09 and 0.16 , respectively. All statistical analyses were performed using Prism software (Graphpad, San Diego, CA).

Simultaneous dual-color time-lapse imaging. Two spectrally distinct fluorophores were simultaneously excited using appropriate filters and then detected using the "Dual-Cam" (Optical-Insights, Tucson, AZ), a device that splits the emission wavelengths into separate (red/green) channels with only minor loss $(\sim 10-15 \%)$ of fluorescence intensity in each channel. Images from each channel were acquired simultaneously by two CoolSnap-HQ cameras (Photometrics, Tucson, AZ). The whole system was run by MetaMorph (Molecular Devices). For dual imaging, only axons showing low and comparable expression of both proteins were analyzed. The videos were then aligned and analyzed as described above.

\section{Results}

\section{Visualization of $\alpha$-synuclein transport in SCb}

In cultured hippocampal neurons, $\alpha$-synuclein first appears at $\sim 7$ DIV and is localized predominantly to presynaptic terminals after establishment of synaptic contacts at $\sim 10-14$ DIV (Withers et al., 1997). Axonal $\alpha$-synuclein was readily identified in cultures double stained for $\alpha$-synuclein and microtubule associated protein 2 (MAP2; a dendritic marker) as MAP2-negative structures that contained punctate $\alpha$-synuclein staining (Fig. $1 A$, arrowheads). Such structures were also identifiable as thin dark processes clearly distinguishable by phase-microscopy from MAP2-positive dendrites and cell bodies (Fig. 1A, arrowheads). We focused on these thin processes to explore $\alpha$-synuclein transport. Neurons were transfected at 6-7 DIV with either mRFP:: $\alpha$-SYN or GFP:: $\alpha-S Y N$, and transport was assessed at 10-15 DIV when endogenous $\alpha$-synuclein is robustly expressed in these cultures. Staining of transfected cultures with the ubiquitous presynaptic marker synaptophysin revealed that the trans- 
A

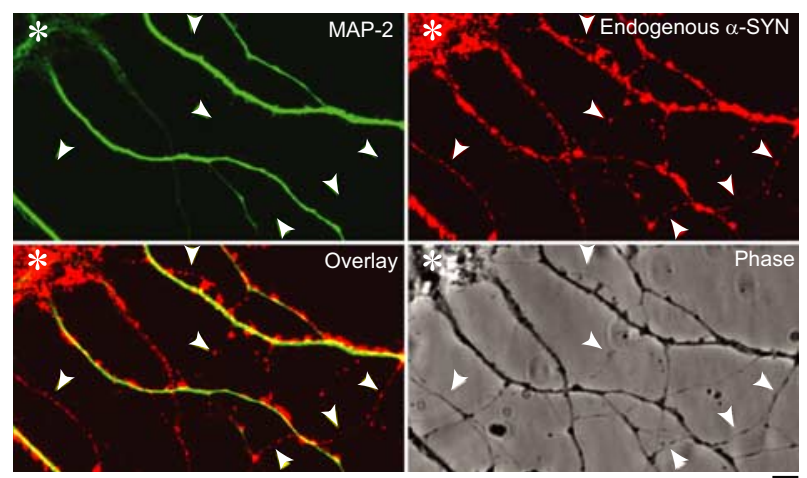

B

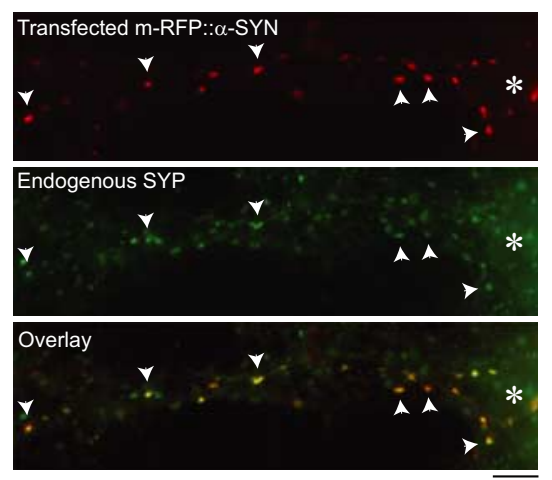

Figure 1. Punctate nonsynaptic localization of endogenous $\alpha$-synuclein particles in axons and characterization of mRFP:: $\alpha$-SYN. $\boldsymbol{A}$, Cultured hippocampal neurons immunostained with MAP2 (green) and $\alpha$-synuclein (red) with corresponding overlay and phase images. Although the bulk of $\alpha$-synuclein localizes to synapses (yellow; along MAP2-positive dendrites in overlay image), punctate nonsynaptic $\alpha$-synuclein (red; arrowheads along MAP2-negative axons) is evident. $\boldsymbol{B}$, Cultured hippocampal neurons transfected with mRFP:: $\alpha$-SYN (red) and subsequently immunostained with the presynaptic marker synaptophysin (green) shows that the bulk of the fusion protein localizes to presynaptic sites (arrowheads), similar to the wild-type protein. Perikarya in $\boldsymbol{A}$ and $\boldsymbol{B}$ are denoted by an asterisk.

fected protein localized prominently to presynaptic boutons, just like endogenous $\alpha$-synuclein (Fig. $1 B$, arrowheads). These results are similar to several other studies indicating that $\mathrm{N}$-terminal tags in $\alpha$-synuclein do not alter biological behavior in mammalian cells (Fortin et al., 2005). In axons, the expressed protein localized to punctate structures. The diffuse mRFP:: $\alpha$-SYN likely represents $\alpha$-synuclein on the plasma membrane and/or in the cytoplasm, consistent with similar reports for several other synaptic proteins (Ahmari et al., 2000). Results obtained with mRFP/GFP tagged fluorescent proteins driven by CMV or synapsin-I promoter were similar (Fig. 1B) (data not shown).

Time-lapse imaging of axonal regions containing fluorescent $\alpha$-synuclein revealed that it was transported both anterogradely and retrogradely (Fig. 2) (supplemental Video 1, available at www.jneurosci.org as supplemental material), with a modest anterograde bias (57\% of the transported particles moved anterogradely). The motility of the transported $\alpha$-synuclein-containing particles in axons was quantified using kymographs as described above. In these kymographs, stationary particles appear as horizontal lines, whereas moving structures appear as diagonal lines, the slopes of which correspond to the average velocity of the moving particles. The kymograph in Figure 2 shows that mRFP:: $\alpha$-SYN puncta are transported in rapid movements (average velocity of the particle was $\sim 1 \mu \mathrm{m} / \mathrm{s}$ during active move- ment) that are interrupted by pauses (e.g., the arrow points to a pause).

To address the possibility that exogenous $\alpha$-synuclein might be transported within the endosomal/lysosomal compartment, as seen occasionally with other exogenously expressed proteins (Roy et al., 2000; Wang and Brown, 2002), we coexpressed mRFP:: $\alpha$-synuclein with GFP::RhoB, a marker of the endosomal/ lysosomal pathway (Wheeler and Ridley, 2004). This enabled both proteins to be visualized simultaneously by dual-color timelapse video microscopy so we could determine the extent to which transported mRFP:: $\alpha$-SYN colocalized with GFP::RhoB. Representative kymographs from these time-lapse experiments are shown in supplemental Fig. S1 (available at www.jneurosci. org as supplemental material). Anterogradely moving $\alpha$-SYN contained almost undetectable levels of GFP::RhoB, suggesting that this material is not within the endosomal/lysosomal compartment but instead reflects $\alpha$-synuclein transport in SCb. Although most retrograde particles were positive for RhoB, $18 \%$ of the particles ( 3 of 17) were negative for the endosomal marker. Therefore, whereas retrograde structures positive for both RhoB and $\alpha$-synuclein probably represents $\alpha$-synuclein moving in degradative pathways, the occasional retrograde mRFP:: $\alpha$-SYN particles negative for RhoB may correspond to a retrograde component of SCb transport. In further support of this, we also observed $5 \%$ of the anterograde $\alpha$-synuclein particles moving bidirectionally ( $>10 \mu \mathrm{m}$ in each direction). We note that a recent study reported movements of GFP:: $\alpha$-SYN in young ( $~ 5$ DIV) cortical cultures (Utton et al., 2005). The movements described in this study were short range $(<5 \mu \mathrm{m})$ and bidirectional, with a retrograde bias, which is very different from our observations of $\alpha$-synuclein transport here showing an anterograde bias commonly spanning much greater distances (see above). In our system, such short-range movements were usually associated with endosomal/lysosomal organelles as evidenced by their colocalization with RhoB during transit. To avoid possible inclusion of $\alpha$-synuclein in endosomal/lysosomal organelles in our analyses of SCb, we only analyzed $\alpha$-synuclein particles that moved at least $10 \mu \mathrm{m}$ (for details, see Materials and Methods).

\section{Direct comparison of $\alpha$-synuclein and} synaptophysin transport

To directly compare the kinetics of SCb to those of FC, we compared $\alpha$-synuclein transport to synaptophysin, a transmembrane protein moving in FC (Elluru et al., 1995). To do this, we used a previously described SYP::GFP fusion protein (Kaether et al., 2000). Synaptophysin is transported in FC as small puncta along axons (Nakata et al., 1998; Kaether et al., 2000), and we obtained comparable results (Fig. 3A) (supplemental Video 2, available at www.jneurosci.org as supplemental material). Comparison of representative kymographs for mRFP:: $\alpha$-SYN and SYP::GFP revealed striking differences in their relative frequencies of active transport (Fig. 3B) (supplemental Videos 3 and 4 and Fig. S2, available at www.jneurosci.org as supplemental material). Although active transport of mRFP:: $\alpha$-SYN is infrequent and interrupted by pauses, SYP::GFP moves nearly continuously with only occasional interruptions over long stretches (typically 30-50 $\mu \mathrm{m})$ of the imaged live axons. However, velocities of the two proteins during episodes of movement are markedly similar. Indeed, as shown by the histograms in Figure $4 A$, the average velocities for all mRFP:: $\alpha$-SYN and SYP::GFP particles are statistically similar during periods of active movement.

Therefore, whereas SYP::GFP moves persistently over long distances, mRFP:: $\alpha$-SYN covers much shorter distances because 
of the frequent pauses in its active translocation, despite moving rapidly during active transport. Presumably, these pauses also make the overall mRFP:: $\alpha$-SYN movements much more infrequent, adding to the overall slow nature of $\alpha$ synuclein transport. Table 1 summarizes the quantitative analyses of mobile anterograde and retrograde mRFP:: $\alpha$-SYN and SYP::GFP particles. It is evident that mRFP:: $\alpha$-SYN moved much less frequently than SYP::GFP, but their anterograde and retrograde transport velocities were similar when they were actively moving. For the above measurements, although all moving mRFP:: $\alpha$-SYN particles could be quantified, it was not feasible to analyze all SYP:GFP particles because of the enormous number of particles that were persistently in motion [see SYP::GFP videos and kymographs in Fig. 3 and supplemental Videos 2 and 4 and Fig. S2 (available at www.jneurosci.org as supplemental material)].

Next, we estimated the proportion of moving versus stationary (paused) mRFP:: $\alpha-S Y N$ and SYP::GFP particles along axons. Here, we analyzed shorter, representative kymographs from both $\alpha$-synuclein and synaptophysin transport experiments to monitor particles that were either moving or stationary. These analyses showed that at any given time, only a small fraction of the anterograde mRFP:: $\alpha$-SYN puncta moved, whereas the majority of puncta were paused in axons (Table 1).

The above data show that although $\alpha$-synuclein and synaptophysin move at comparable velocities during active transport, their overall transport behavior is very different. This in turn suggests that they move completely separately from each other. To directly test this possibility, we visualized the transport of mRFP:: $\alpha$-SYN and GFP::SYP in the same axons using simultaneous live-cell dual-color video microscopy. Figure 5, $A-D$, shows selected frames and kymographs from these experiments (supplemental Video 5, available at www.jneurosci.org as supplemental material), which indicate that at any given time, many more SYP::GFP particles move compared with mRFP:: $\alpha$-SYN particles. Because the density of transported SYP::GFP particles is high and their movements frequently overlap, the exact number of particles cannot be determined, but during a $4475 \mathrm{~s}$ imaging period, there were several hundred particles. In comparison, we only observed 22 moving $\alpha$-synuclein particles. These data show in a very direct manner that the vast majority of the transported synaptophysin moves independently from $\alpha$-synuclein, albeit not exclusively independently, because we observed instances in which synaptophysin and $\alpha$-synuclein were cotransported. In some examples, synaptophysin and $\alpha$-synuclein moved together throughout the video record (Fig. 5D, yellow line in merged image), whereas in other cases, particles of $\alpha$-synuclein and synaptophysin appeared to merge and move together transiently for short distances (Fig. $5 B, C$, yellow lines in merged images). These results may correspond to in vivo radiolabeling studies, which show that a small fraction of total labeled synaptophysin could be detected at times when $\mathrm{SCb}$ was the main fraction of radiolabeled protein in the nerve segment (Elluru et al., 1995). Also, cofrac-
mRFP:: $\alpha-S Y N$ time-lapse imaging
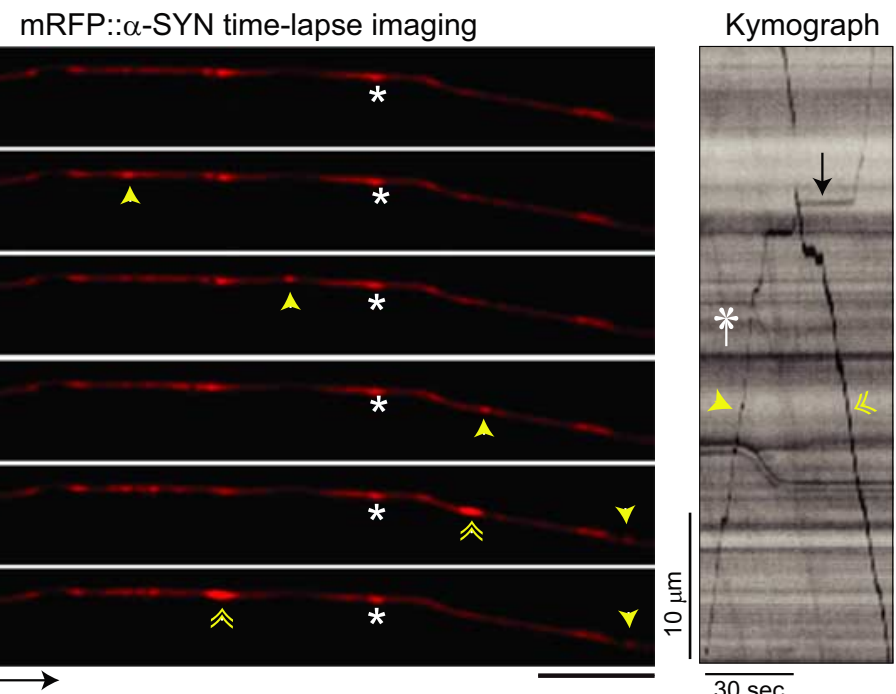

$30 \mathrm{sec}$

Figure 2. Punctate $\alpha$-synuclein particles are transported bidirectionally in axons. $\boldsymbol{A}$, Axonal transport of transfected mRFP:: $\alpha$-SYN particles in 10-14 DIV hippocampal neurons. Selected video frames show mRFP:: $\alpha$-SYN particles transported ( the video frames to the left. Note that the movement is rapid, with pauses (arrow) during transit. Scale bars for the kymographs are (s). Scale bar, $5 \mu \mathrm{m}$.

tionation of $\alpha$-synuclein with membrane fractions that include synaptophysin has been demonstrated in mouse and human brains (Kahle et al., 2000). It is also well established that $\alpha$-synuclein can bind to membranes under certain conditions (Kubo et al., 2005). Therefore, the limited cotransport that we observed may reflect similar interactions occurring in the axon.

\section{Cotransport of $\mathrm{SCb}$ proteins}

To gain additional insights into the underlying mechanisms of $\mathrm{SCb}$ transport, we asked whether other SCb proteins also moved in a rapid but infrequent manner and whether different $\mathrm{SCb}$ proteins are transported independently of each other as distinct cargoes or transported together. To accomplish this, we transfected cultured hippocampal neurons with $\alpha$-synuclein and two different $\mathrm{SCb}$ proteins and visualized them simultaneously using the dual-cam system we developed. The first SCb protein we chose was synapsin-I, and like $\alpha$-synuclein, it is also a vesicle-associated protein enriched at presynapses. In addition, we studied an unrelated protein that also moves in SCb. Therefore, the second $\mathrm{SCb}$ protein we chose was GAPDH, a glycolytic enzyme localized throughout the axons. Both synapsin-I and GAPDH are established SCb proteins (Baitinger and Willard, 1987; Paggi and Petrucci, 1992; Yuan et al., 1999).

For these experiments, we transfected mRFP:: $\alpha$-SYN with either synapsin-I or GAPDH tagged with GFP (GFP::SYS-I and GFP::GAPDH, respectively) in cultured hippocampal neurons and visualized the red/green fluorophores simultaneously using our dual-cam system. Simultaneous visualization of the red/ green fusion proteins during transport showed that most mRFP:: $\alpha$-SYN particles were cotransported both anterogradely and retrogradely with GFP::SYS-I and GFP::GAPDH (Fig. 6A-F) (supplemental Videos 6 and 7, available at www.jneurosci.org as supplemental material). Representative kymographs clearly demonstrate the cotransport of the mRFP:: $\alpha-S Y N$ with GFP::SYS-1 (Fig. 6B,C) and mRFP:: $\alpha$-SYN with GFP::GAPDH 
A
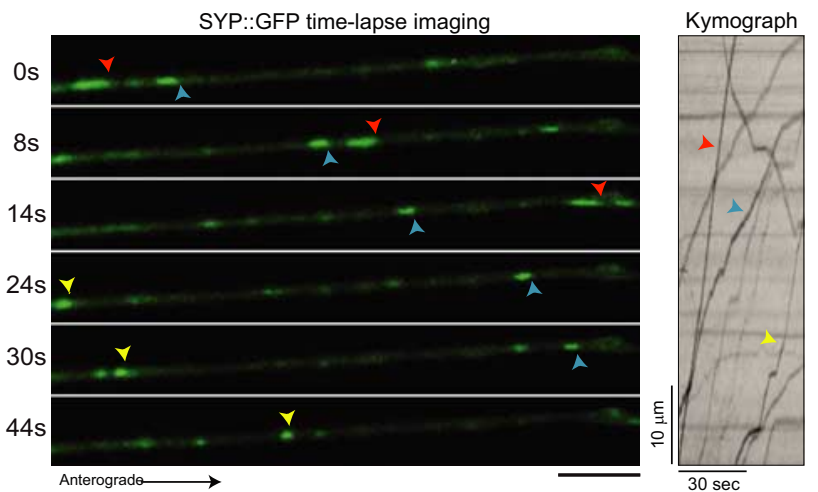

B

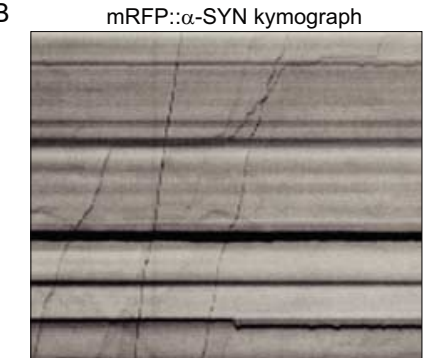

SYP::GFP kymograph
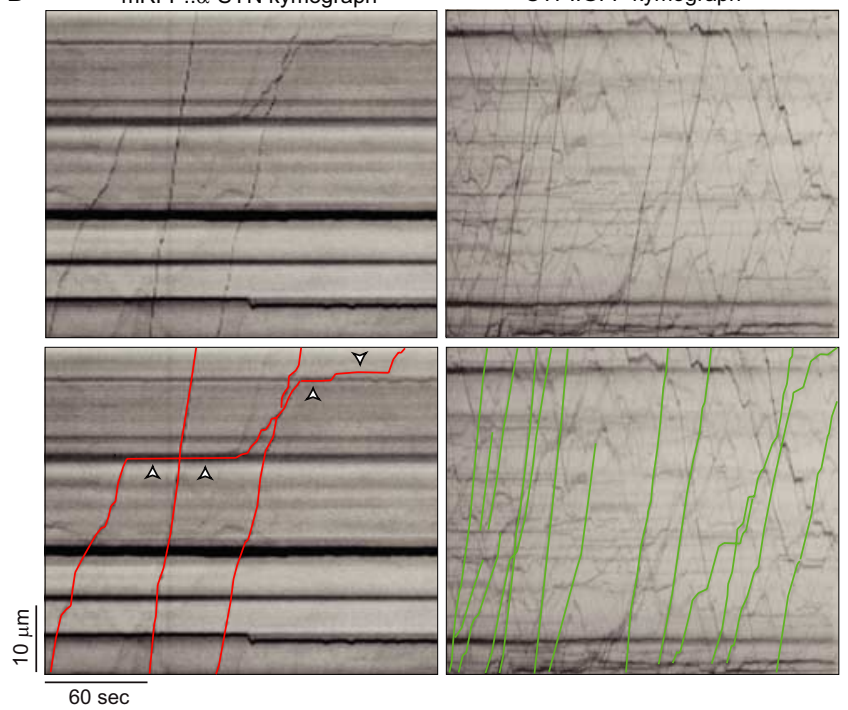

Figure 3. Axonal transport dynamics of synaptophysin compared with those of $\alpha$-synuclein A, Axonal transport of SYP::GFP particles in cultured hippocampal neurons. Several anterograde particles are observed, three of which are marked by colored arrowheads (red, blue, and yellow). The corresponding kymograph is shown on the right. Lines on the kymograph marked by colored arrowheads represent transport of similarly marked particles in the video frames to the left. (For the corresponding video, see supplemental video 2 , available at www.jneurosci.org as supplemental material.) $\boldsymbol{B}$, Representative kymographs obtained from mRFP:: $\alpha$-SYN (left) and SYP::GFP (right) imaging. In the kymographs below, anterogradely moving particles are overlaid with red or green lines representing movement of $\alpha$-synuclein and synaptophysin, respectively. Note the infrequent and rapid mRFP::. $\alpha$-SYN movements, interrupted by pauses (arrowheads), unlike the frequent, rapid, and persistent movements of SYP::GFP. (For videos corresponding to these kymographs, see supplemental videos 3 and 4, available at www. jneurosci.org as supplemental material.) Scale bars for the kymographs are at the bottom left of kymograph(s). Scale bar, $5 \mu \mathrm{m}$.

(Fig. 6E,F) over long distances. Quantitative analyses of these experiments (Table 2) show that both synapsin-I and GAPDH are transported rapidly but infrequently in axons, similar to $\alpha$-synuclein, and that most synapsin-I and GAPDH particles are cotransported with $\alpha$-synuclein.

\section{Discussion}

This study reveals novel mechanistic details about transport of $\mathrm{SCb}$ proteins. We showed that SCb proteins move rapidly at velocities similar to those seen in fast transport, but the movements are infrequent and individual particles often pause, leading to a slow overall rate when averaged over time. Additionally, using simultaneous dual-color video microscopy, we showed that $\mathrm{SCb}$ transport is distinct from the FC, and that multiple SCb proteins are cotransported in axons, suggesting that they move as compo-
Histogram of average velocities

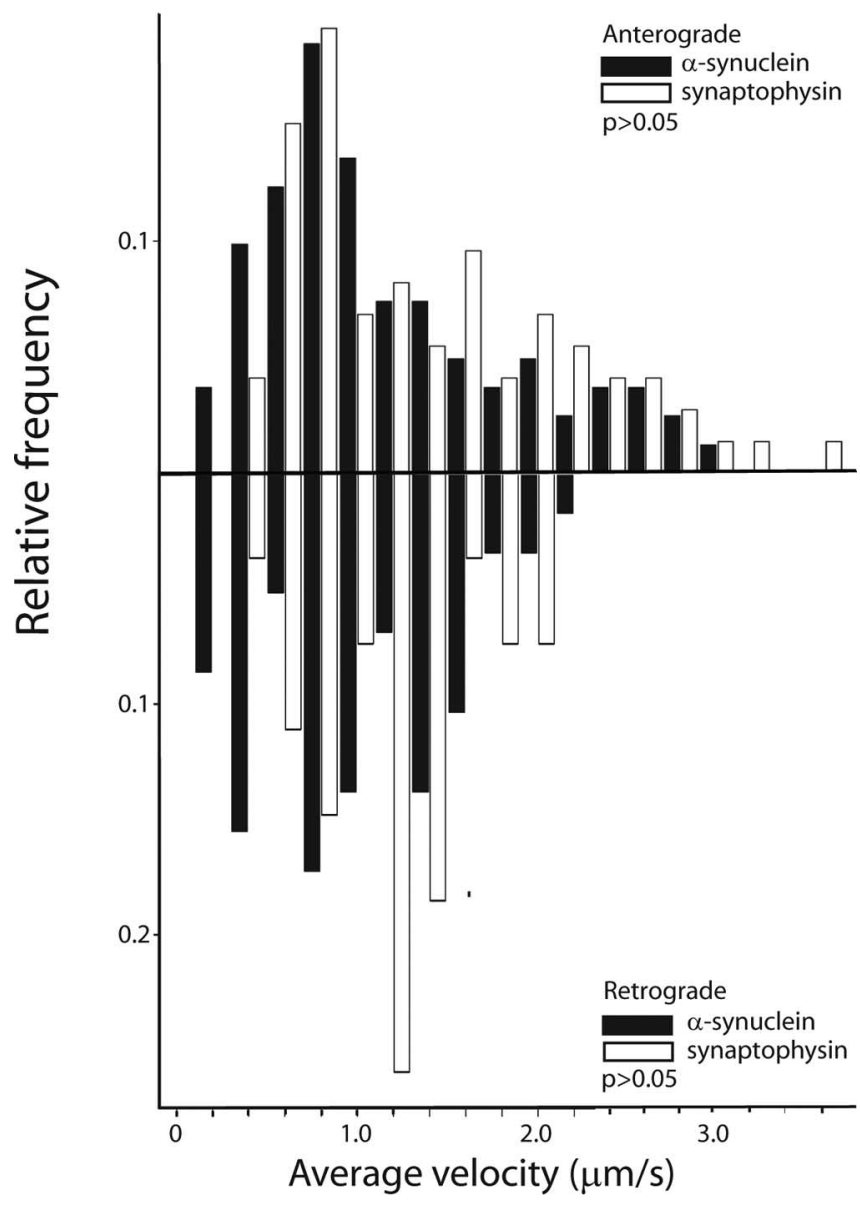

Figure 4. Frequency histograms of transported $\alpha$-synuclein and synaptophysin. $\boldsymbol{A}$, Frequency histograms of average anterograde and retrograde velocities of moving mRFP:: $\alpha$-SYN and SYP:GFP particles (excluding pauses) show that they both move rapidly at a broad range of velocities, and the groups are statistically similar ( $p>0.05$; Mann-Whitney test, two tailed).

nents of common cargoes. The kinetics of SCb transport in our study explains for the first time some of the unique features of this rate-class, whereas our data on the cotransport of SCb proteins provides new insights on the nature of SCb cargoes.

\section{Rapid, intermittent transport of $\mathrm{SCb}$ proteins}

When neurofilaments and microtubules moving in SCa were visualized by live-cell imaging (Roy et al., 2000; Wang et al., 2000; Wang and Brown, 2002), it was shown that cytoskeletal proteins were transported rapidly and infrequently but with significant pauses during transit. These and other findings led to the "stop and go" hypothesis (Brown, 2003; Brown et al., 2005), which proposes that the overall slower rate of the slow component population is an average of rapid movements of individual cargoes interrupted by pauses. Although these studies advance understanding of SCa transport mechanisms, SCb mechanisms remained unclear. Therefore, our data are significant, because we show here that even noncytoskeletal proteins in $\mathrm{SCb}$ move in a stop and go manner but with several differences as discussed below.

Although both SCa and SCb proteins are transported rapidly but infrequently, their overall rates as determined by radiolabeling studies differ by a factor of $\sim 2-10$ (Brown, 2003). These differences can be ascribed to differences in the intrinsic properties of the motors, the frequency of transport, physical properties 
of the cargoes that influence the movement, or any combination of the above factors. The available data from cultured sympathetic and hippocampal neurons show that average rates of $\alpha$-synuclein particles transported in $\mathrm{SCb}(\sim 1 \mu \mathrm{m} / \mathrm{s})$ are faster than the rates of neurofilaments moving in SCa $(\sim 0.4-0.7 \mu \mathrm{m} / \mathrm{s})$. The frequency of anterograde SCb transport in our study (one movement every $\sim 2 \mathrm{~min}$ ) is also higher than the frequency of anterograde neurofilament transport in SCa (one movement every $\sim 3.5-5 \mathrm{~min}$ ). The predominantly cytosolic cargoes of $\mathrm{SCb}$ are also very different from the cytoskeletal cargoes in SCa. Therefore, any or all of the differences in kinetics of SCa and SCb seen in the live-cell studies could account for the generation of the faster SCb rates. In accordance with the higher transport frequency of $\mathrm{SCb}$ when compared with neurofilaments, it is also our impression that SCb proteins seem to move greater distances during episodes of active transport. For instance, although SCb particles spent the majority of their time pausing and the frequency of movement is obviously much lower than the FC protein synaptophysin, we observed several examples of transport in which $\alpha$-synuclein particles moved almost continuously throughout the time imaged [see $\alpha$-synuclein kymographs in Fig. 3 and in supplemental Fig. 1 (available at www. jneurosci.org as supplemental material)].

\section{Cotransport of cargoes: a common theme in axonal transport}

Although cargoes moving in the FC are mainly associated with vesicles, and those in SCa are cytoskeletal, SCb cargoes are a diverse and primarily unrelated group of proteins moving together in axons. How are these $\geq 200$ proteins with a variety of biophysical properties moving in axons at fairly discrete overall rates? Our observation that both synaptic and nonsynaptic $\mathrm{SCb}$ proteins are transported together raises the possibility that they move in common carriers and/or as constituents of common cargoes, perhaps as multiprotein complexes transported within axons. Although the precise nature of these complexes is unknown, our study suggests that at least one such complex could contain the three SCb proteins: $\alpha$-synuclein, synapsin-I, and GAPDH.

Cotransport of multiprotein complexes has been observed in fast axonal transport of presynaptic proteins as well, and two biochemically and morphologically distinct organelles have been identified. Whereas the transported synaptic vesicles move in a multivesicular complex comprised of several different proteins called synaptic-vesicle protein transport vesicle (STV) (Ahmari et al., 2000), the active zone components are thought to be transported separately in dense-core vesicular structures called "pic-
Table 1. Quantitative analysis of $\alpha$-synuclein and synaptophysin transport

\begin{tabular}{lllllc}
\hline & Direction & $\begin{array}{l}\text { Number of } \\
\text { moving particles }\end{array}$ & $\begin{array}{l}\text { Frequency of } \\
\text { movement }(\# / \mathrm{s})\end{array}$ & $\begin{array}{l}\text { Average velocity } \\
(\mu \mathrm{m} / \mathrm{s})\end{array}$ & $\begin{array}{r}\text { Estimated \% of } \\
\text { moving objects }\end{array}$ \\
\hline \multirow{2}{*}{$\alpha$-Synuclein } & Anterograde & 81 & $1 / 120$ & $1.18 \pm 0.08$ & 7.6 \\
& Retrograde & 62 & $1 / 157$ & $0.99 \pm 0.09$ & 3.5 \\
Synaptophysin & Anterograde & 56 & $1 / 10$ & $1.40 \pm 0.07$ & 32.6 \\
& Retrograde & 22 & $1 / 26$ & $1.16 \pm 0.08$ & 13.3 \\
\hline
\end{tabular}

Quantitative analysis of mRFP:: $\alpha$-SYN and SYP::GFP particles are shown. All data are derived from kymograph analysis, and synaptophysin movements were analyzed as described in Materials and Methods. Average velocities (when moving) are expressed as mean \pm SEM. The proportion of moving objects was estimated as described in Materials and Methods.

A mRFP:: $\alpha-S Y N$ SYP::GFP
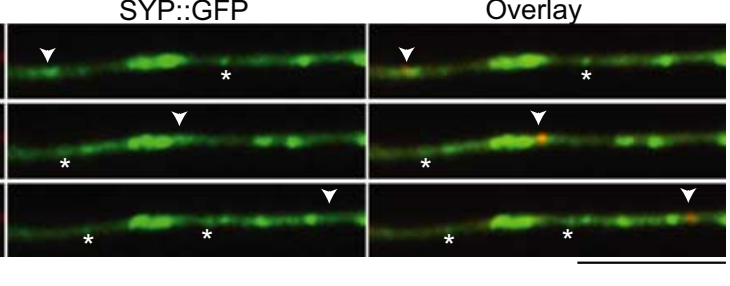

B
mRFP:: $\alpha$-syn
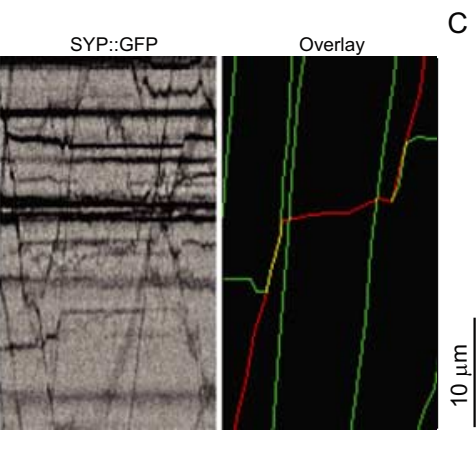

C

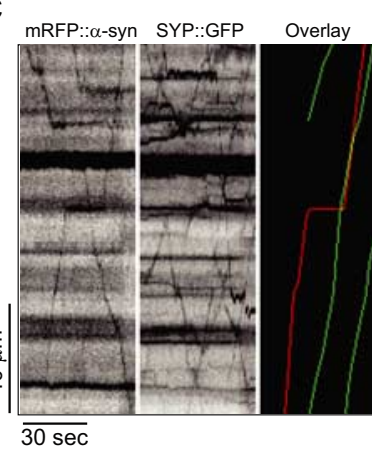

$\mathrm{D}$
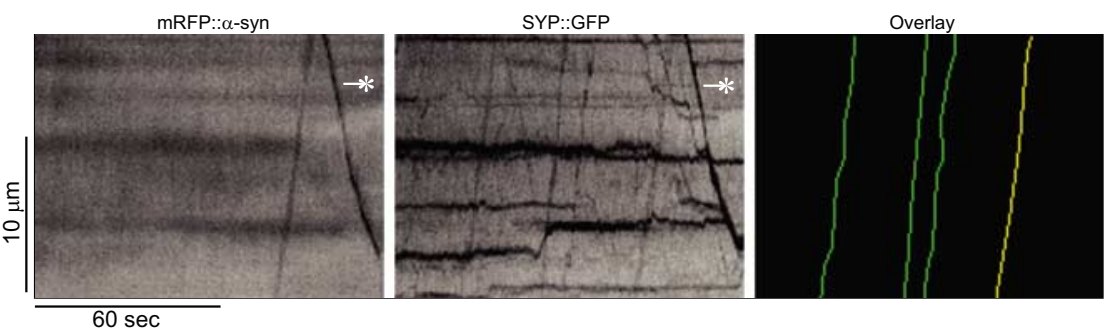

Figure 5. Simultaneous visualization of transport kinetics of $\alpha$-synuclein and synaptophysin in the same axon. Cultured hippocampal neurons were double-transfected with mRFP:: $\alpha$-SYN and SYP.:GFP and visualized by simultaneous dual-color video microscopy. $\boldsymbol{A}$, Selected frames from a time-lapse sequence shows a single mRFP.: $\alpha$-SYN particle (red) transported anterogradely (arrowhead). In the same axon, several SYP::GFP particles (green) are seen, including those being transported (marked with asterisks, also see the corresponding kymograph). (For corresponding video, see supplemental Video 5, available at www. neurosci.org as supplemental material.) $\boldsymbol{B}-\boldsymbol{D}$, Kymographs from dual-cam experiments wherein each three-frame panel shows (mRFP:: $\alpha$-SYN in red and SYP::GFP in green) from the two kymographs (right). The kymograph in $\boldsymbol{B}$ is from the time-lapse shown above. Note the different transport frequencies and cotransport (yellow diagonal lines in merged kymographs) of mRFP:: $\alpha$-SYN and SYP:GFP. Retrograde mRFP:: $\alpha$-SYN particles were often cotransported with SYP.:GFP; the asterisk in $\boldsymbol{D}$ marks one such particle. Scale bars for the kymographs are at the bottom left of the kymograph(s). Scale bar, $5 \mu \mathrm{m}$.

colo transport vesicles" (PTV) (Zhai et al., 2001; Shapira et al., 2003). Thus, the STVs may help in synaptic vesicular complex organization the PTVs may help consolidate the active zones at presynaptic terminals. Cotransport has also been observed for scaffolding proteins and elements of the signaling pathways (Verhey et al., 2001). This kind of efficient "packaging" of multiple polypeptides may help them perform their independent but complementary roles at synaptic terminals (Sytnyk et al., 2004) and may represent a common theme in intracellular transport of fast and slow component proteins (Almenar-Queralt and Goldstein, 2001). 


\section{Simultaneous dual-cam imaging of mRFP:: $\alpha-S Y N$ and GFP::SYS-I}

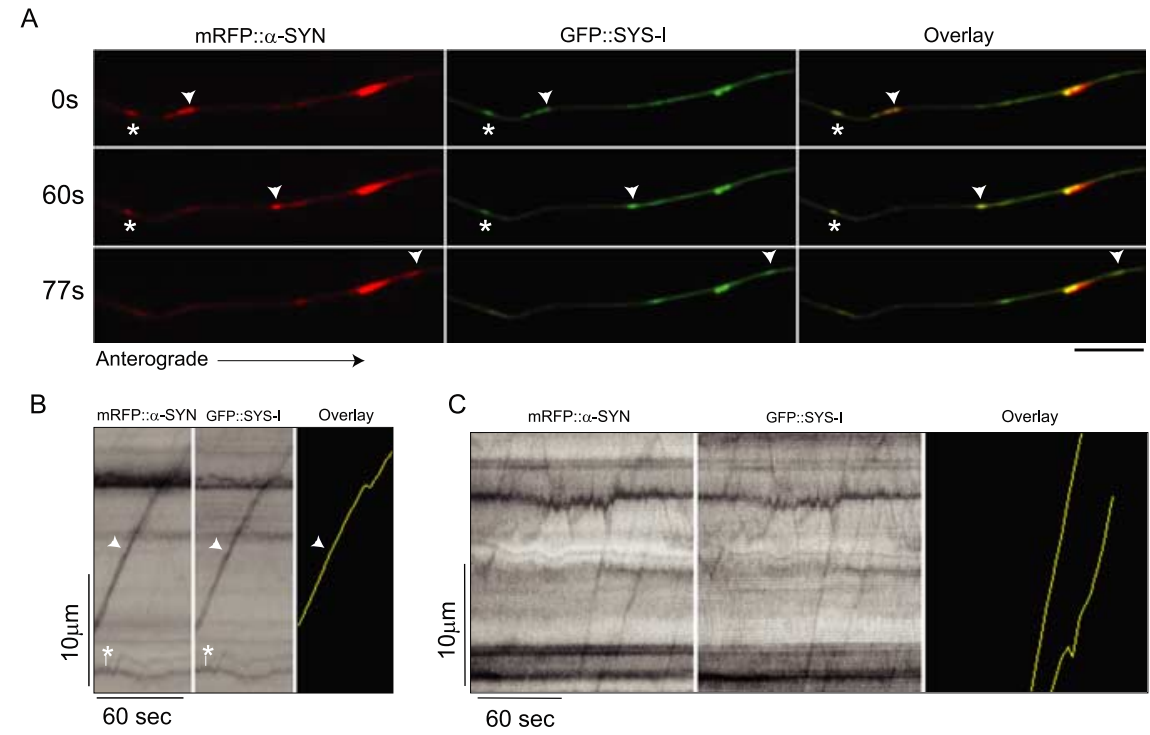

Simultaneous dual-cam imaging of mRFP:: $\alpha-S Y N$ and GFP::GAPDH

D

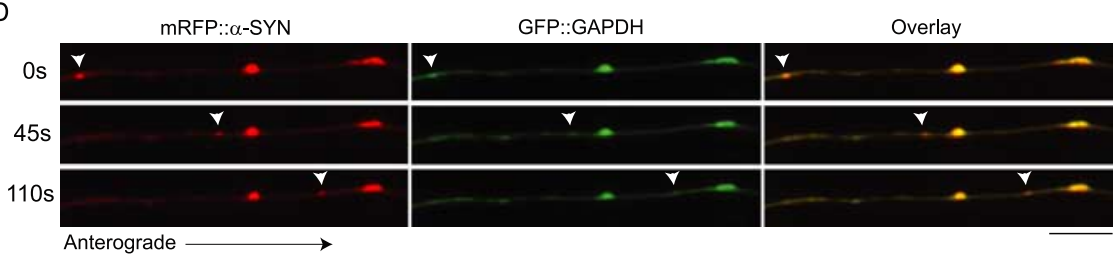

$E$

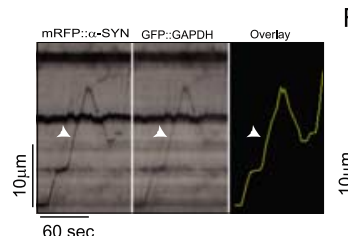

$\mathrm{F}$
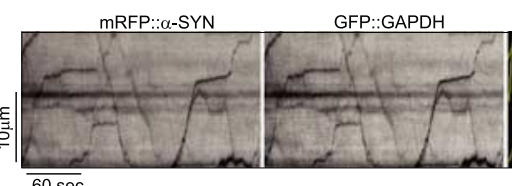

Figure 6. Simultaneous visualization of SCb proteins reveal that they are cotransported anterogradely. Cultured hippocampal neurons were double transfected with mRFP:: $\alpha$-SYN and either GFP::SYS-I or GFP::GAPDH (all SCb proteins) and visualized by simultaneous dual-color video microscopy. $\boldsymbol{A}-\boldsymbol{F}$ show selected frames and kymographs from dual-cam imaging. Each set of frames shows $\alpha$-synuclein (red) and synapsin-I or GAPDH (green), with the corresponding overlay image (yellow). Each three-frame kymograph panel shows the mRFP:: $\alpha$-SYN kymograph (left), GFP::SYS-I or GFP::GAPDH kymograph (middle), and a pseudocolor overlay of anterograde tracings from the respective kymographs (right). The yellow lines in overlaid kymographs represent cotransport. $\boldsymbol{A}, \boldsymbol{B}$, Cotransport of an object (arrowhead) positive for mRFP:: $\alpha$-SYN and GFP::SYS-I; the asterisk marks a stationary object. Arrowheads and asterisks in $\boldsymbol{B}$ represent movement of correspondingly marked particles in the frames above. $\boldsymbol{C}$, Additional kymograph showing cotransport and pausing of an anterograde mRFP:: $\alpha$-SYN/GFP::SYS-I object. $\boldsymbol{D}, \boldsymbol{E}$, Cotransport of an object (arrowhead) positive for mRFP:: $\alpha$-SYN and GFP::GAPDH with corresponding kymographs. $\boldsymbol{F}$, Additional examples of mRFP:: $\alpha$-SYN/GFP::GAPDH cotransport. (For corresponding videos, see supplemental videos 6 and 7 , available at www.jneurosci. org as supplemental material.) Scale bars for the kymographs are at the bottom left of the kymograph(s). Scale bar, $5 \mu \mathrm{m}$.

Table 2. Quantitative analysis of simultaneous visualization of SCb proteins

\begin{tabular}{lllll}
\hline & $\begin{array}{l}\text { Number of } \\
\text { moving particles }\end{array}$ & $\begin{array}{l}\text { Frequency of } \\
\text { movement }(\# / s)\end{array}$ & $\begin{array}{l}\text { Average velocity } \\
(\mu \mathrm{m} / \mathrm{s})\end{array}$ & $\begin{array}{l}\text { Particles cotransported with } \\
\alpha \text {-synuclein }\end{array}$ \\
\hline Synapsin-I & 31 & $1 / 181$ & $0.98 \pm 0.09$ & $27 / 31(87 \%)$ \\
GAPDH & 29 & $1 / 180$ & $1.18 \pm 0.13$ & $26 / 29(86 \%)$ \\
\hline
\end{tabular}

All data were obtained from kymograph analysis. Frequency and average velocities of the SCb proteins synapsin-I and GAPDH were similar to $\alpha$-synuclein. Average velocities (when moving) are expressed as mean \pm SEM. The majority of the synapsin-I and GAPDH particles were also cotransported with $\alpha$-synuclein.

The idea of "SCb complexes" was originally proposed by classical radiolabeling studies that noted a fairly coherent transport of the front of the radiolabeled SCb "wave" (Garner and Lasek, 1982 ) and is also supported by experiments showing that $\mathrm{SCb}$

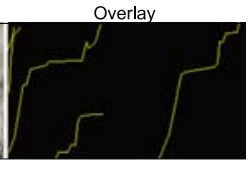

components can be coimmunoprecipitated from cultured axons (Black et al., 1991). Although such a cotransport was also proposed for SCa (Lasek et al., 1984), recent motor inhibition studies strongly suggest that the two SCa components neurofilaments and microtubules use distinct transport mechanisms (Xia et al., 2003; He et al., 2005). However, in contrast to SCa, our direct observations suggest that $\mathrm{SCb}$ proteins indeed move together in axons. One question is whether all $\mathrm{SCb}$ proteins are transported as a single complex or multiple $\mathrm{SCb}$ complexes exist in axons. Although we examined only three SCb proteins, our data suggest the latter scenario, because not all synapsin-I and GAPDH particles were cotransported, possibly because they are also transported as components of other, yet undefined complexes. Therefore, $\mathrm{SCb}$ appears to represent a heterogeneous group of cargo complexes that exhibit comparable transport kinetics, much as the FC represents the movement of multiple vesicle populations moving with similar kinetics.

In summary, our results reveal fundamental aspects of $\mathrm{SCb}$, derived from direct observations of axonal transport in cultured neurons. We show that three $\mathrm{SCb}$ proteins are transported infrequently and intermittently, unlike synaptophysin transported in FC. By simultaneous visualization of slow and fast component proteins, we show that synaptophysin transport is primarily independent of $\alpha$-synuclein, and that various $\mathrm{SCb}$ proteins show almost identical kinetics during transport, suggesting that they may be transported as components of common cargos. We anticipate that our model system for the study of SCb transport will lead to additional insights into basic mechanisms of this component of axonal transport. Additionally, because defective axonal transport of $\alpha$-synuclein has been implicated in the pathogenesis of Lewy bodies and brain degeneration in Parkinson's disease (PD) (Jensen et al., 1999; Roy et al., 2005), our observations of $\alpha$-synuclein transport in SCb open up new avenues for investigating the role of altered $\alpha$-synuclein transport in mechanisms of PD.

\section{References}

Ahmari SE, Buchanan J, Smith SJ (2000) Assembly of presynaptic active zones from cytoplasmic transport packets. Nat Neurosci 3:445-451.

Almenar-Queralt A, Goldstein LS (2001) Linkers, packages and pathways: new concepts in axonal transport. Curr Opin Neurobiol 11:550-557.

Baas PW, Buster DW (2004) Slow axonal transport and the genesis of neuronal morphology. J Neurobiol 58:3-17. 
Baitinger C, Willard M (1987) Axonal transport of synapsin I-like proteins in rabbit retinal ganglion cells. J Neurosci 7:3723-3735.

Black MM, Lasek RJ (1980) Slow components of axonal transport: two cytoskeletal networks. J Cell Biol 86:616-623.

Black MM, Chestnut MH, Pleasure IT, Keen JH (1991) Stable clathrin: uncoating protein (HSC70) complexes in intact neurons and their axonal transport. J Neurosci 11:1163-1172.

Borchelt DR, Wong PC, Becher MW, Pardo CA, Lee MK, Xu ZS, Thinakaran G, Jenkins NA, Copeland NG, Sisodia SS, Cleveland DW, Price DL, Hoffman PN (1998) Axonal transport of mutant superoxide dismutase 1 and focal axonal abnormalities in the proximal axons of transgenic mice. Neurobiol Dis 5:27-35.

Bourke GJ, El Alami W, Wilson SJ, Yuan A, Roobol A, Carden MJ (2002) Slow axonal transport of the cytosolic chaperonin CCT with Hsc73 and actin in motor neurons. J Neurosci Res 68:29-35.

Bray JJ, Fernyhough P, Bamburg JR, Bray D (1992) Actin depolymerizing factor is a component of slow axonal transport. J Neurochem 58:2081-2087.

Brown A (2003) Axonal transport of membranous and nonmembranous cargoes: a unified perspective. J Cell Biol 160:817-821.

Brown A, Wang L, Jung P (2005) Stochastic simulation of neurofilament transport in axons: the "stop-and-go" hypothesis. Mol Biol Cell 16:4243-4255.

Dillman III JF, Dabney LP, Pfister KK (1996) Cytoplasmic dynein is associated with slow axonal transport. Proc Natl Acad Sci USA 93:141-144.

Elluru RG, Bloom GS, Brady ST (1995) Fast axonal transport of kinesin in the rat visual system: functionality of kinesin heavy chain isoforms. Mol Biol Cell 6:21-40.

Forman MS, Trojanowski JQ, Lee VM (2004) Neurodegenerative diseases: a decade of discoveries paves the way for therapeutic breakthroughs. Nat Med 10:1055-1063.

Fortin DL, Nemani VM, Voglmaier SM, Anthony MD, Ryan TA, Edwards RH (2005) Neural activity controls the synaptic accumulation of $\alpha$-synuclein. J Neurosci 25:10913-10921.

Garner JA, Lasek RJ (1982) Cohesive axonal transport of the slow component b complex of polypeptides. J Neurosci 2:1824-1835.

Hafezparast M, Klocke R, Ruhrberg C, Marquardt A, Ahmad-Annuar A, Bowen S, Lalli G, Witherden AS, Hummerich H, Nicholson S, Morgan PJ, Oozageer R, Priestley JV, Averill S, King VR, Ball S, Peters J, Toda T, Yamamoto A, Hiraoka Y, et al. (2003) Mutations in dynein link motor neuron degeneration to defects in retrograde transport. Science 300:808-812.

He Y, Francis F, Myers KA, Yu W, Black MM, Baas PW (2005) Role of cytoplasmic dynein in the axonal transport of microtubules and neurofilaments. J Cell Biol 168:697-703.

Jacob JM, McQuarrie IG (1991) Axotomy accelerates slow component b of axonal transport. J Neurobiol 22:570-582.

Jensen PH, Li JY, Dahlstrom A, Dotti CG (1999) Axonal transport of synucleins is mediated by all rate components. Eur J Neurosci 11:3369-3376.

Kaether C, Skehel P, Dotti CG (2000) Axonal membrane proteins are transported in distinct carriers: a two-color video microscopy study in cultured hippocampal neurons. Mol Biol Cell 11:1213-1224.

Kahle PJ, Neumann M, Ozmen L, Muller V, Jacobsen H, Schindzielorz A, Okochi M, Leimer U, van der PH, Probst A, Kremmer E, Kretzschmar HA, Haass C (2000) Subcellular localization of wild-type and Parkinson's disease-associated mutant $\alpha$-synuclein in human and transgenic mouse brain. J Neurosci 20:6365-6373.
Kubo S, Nemani VM, Chalkley RJ, Anthony MD, Hattori N, Mizuno Y, Edwards RH, Fortin DL (2005) A combinatorial code for the interaction of alpha-synuclein with membranes. J Biol Chem 280:31664-31672.

Lasek RJ, Garner JA, Brady ST (1984) Axonal transport of the cytoplasmic matrix. J Cell Biol 99:212s-221s.

Maier CE, McQuarrie IG (1990) Increased slow transport in axons of regenerating newt limbs after a nerve conditioning lesion. Dev Biol 140:172-181.

Nakata T, Terada S, Hirokawa N (1998) Visualization of the dynamics of synaptic vesicle and plasma membrane proteins in living axons. J Cell Biol 140:659-674.

Paggi P, Petrucci TC (1992) Neuronal compartments and axonal transport of synapsin I. Mol Neurobiol 6:239-251.

Roy S, Coffee P, Smith G, Liem RK, Brady ST, Black MM (2000) Neurofilaments are transported rapidly but intermittently in axons: implications for slow axonal transport. J Neurosci 20:6849-6861.

Roy S, Zhang B, Lee VM, Trojanowski JQ (2005) Axonal transport defects: a common theme in neurodegenerative diseases. Acta Neuropathol (Berl) 109:5-13.

Saha AR, Hill J, Utton MA, Asuni AA, Ackerley S, Grierson AJ, Miller CC, Davies AM, Buchman VL, Anderton BH, Hanger DP (2004) Parkinson's disease alpha-synuclein mutations exhibit defective axonal transport in cultured neurons. J Cell Sci 117:1017-1024.

Shapira M, Zhai RG, Dresbach T, Bresler T, Torres VI, Gundelfinger ED, Ziv NE, Garner CC (2003) Unitary assembly of presynaptic active zones from Piccolo-Bassoon transport vesicles. Neuron 38:237-252.

Sytnyk V, Leshchyns'ka I, Dityatev A, Schachner M (2004) Trans-Golgi network delivery of synaptic proteins in synaptogenesis. J Cell Sci 117:381-388.

Utton MA, Noble WJ, Hill JE, Anderton BH, Hanger DP (2005) Molecular motors implicated in the axonal transport of tau and alpha-synuclein. J Cell Sci 118:4645-4654.

Verhey KJ, Meyer D, Deehan R, Blenis J, Schnapp BJ, Rapoport TA, Margolis B (2001) Cargo of kinesin identified as JIP scaffolding proteins and associated signaling molecules. J Cell Biol 152:959-970.

Wang L, Brown A (2002) Rapid movement of microtubules in axons. Curr Biol 12:1496-1501.

Wang L, Ho CL, Sun D, Liem RK, Brown A (2000) Rapid movement of axonal neurofilaments interrupted by prolonged pauses. Nat Cell Biol 2:137-141.

Wheeler AP, Ridley AJ (2004) Why three Rho proteins? RhoA, RhoB, RhoC, and cell motility. Exp Cell Res 301:43-49.

Withers GS, George JM, Banker GA, Clayton DF (1997) Delayed localization of synelfin (synuclein, NACP) to presynaptic terminals in cultured rat hippocampal neurons. Brain Res Dev Brain Res 99:87-94.

Wujek JR, Lasek RJ (1983) Correlation of axonal regeneration and slow component B in two branches of a single axon. J Neurosci 3:243-251.

Xia CH, Roberts EA, Her LS, Liu X, Williams DS, Cleveland DW, Goldstein LS (2003) Abnormal neurofilament transport caused by targeted disruption of neuronal kinesin heavy chain KIF5A. J Cell Biol 161:55-66.

Yuan A, Mills RG, Bamburg JR, Bray JJ (1999) Cotransport of glyceraldehyde-3-phosphate dehydrogenase and actin in axons of chicken motoneurons. Cell Mol Neurobiol 19:733-744.

Zhai RG, Vardinon-Friedman H, Cases-Langhoff C, Becker B, Gundelfinger ED, Ziv NE, Garner CC (2001) Assembling the presynaptic active zone: a characterization of an active one precursor vesicle. Neuron 29:131-143. 